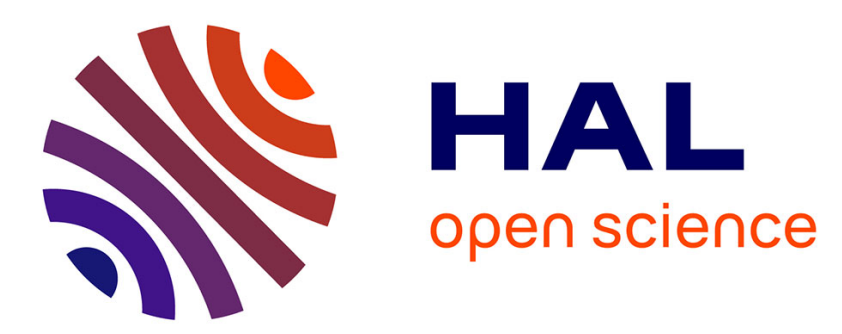

\title{
A-Translation and Looping Combinators in Pure Type Systems
}

Thierry Coquand, Hugo Herbelin

\section{To cite this version:}

Thierry Coquand, Hugo Herbelin. A-Translation and Looping Combinators in Pure Type Systems. Journal of Functional Programming, 1994, 4 (1), pp.77-88. inria-00159085

\section{HAL Id: inria-00159085 https://hal.inria.fr/inria-00159085}

Submitted on 2 Jul 2007

HAL is a multi-disciplinary open access archive for the deposit and dissemination of scientific research documents, whether they are published or not. The documents may come from teaching and research institutions in France or abroad, or from public or private research centers.
L'archive ouverte pluridisciplinaire HAL, est destinée au dépôt et à la diffusion de documents scientifiques de niveau recherche, publiés ou non, émanant des établissements d'enseignement et de recherche français ou étrangers, des laboratoires publics ou privés. 


\title{
$A$-translation and Looping Combinators in Pure Type Systems
}

\author{
Thierry Coquand, coquand@cs.chalmers.se
}

Hugo Herbelin, herbelin@margaux.inria.fr

\begin{abstract}
We present here a generalization of $A$-translation to a class of Pure Type Systems.

We apply this translation to give a direct proof of the existence of a looping combinator in a large class of inconsistent type systems, class which includes type systems with a type of all types. This is the first non-automated solution to this problem.
\end{abstract}

\section{Introduction}

The term A-translation first appeared in a paper of Friedman [3]. It denotes there a technical tool used in a proof of closure under Markov's rule of several intuitionistic systems. Combined with Gödel's translation from classical arithmetic into intuitionistic arithmetic, this was used to give a new proof of the intuitionistic provability of classically provable $\Sigma_{1}^{0}$ formulas.

Leivant [8] is a good reference about $A$-translation. Recently, connections between $A$-translation and Continuation Passing Style have been investigated. See for instance Murthy's Ph. D. thesis[10].

We are going to generalise $A$-translation to a large class of Pure Type Systems, introduced recently by Barendregt $[1,4]$. This generalisation is motivated by the following problem: to extract constructive informations from paradoxes in inconsistent type systems. More specifically, let us define a "looping combinator" as being a term having the same Böhm tree as the fixed-point combinator $Y$. It has been shown by Howe [6] that a type system with a type of all types contain a looping combinator. We will get this result as an application of $A$ translation for Pure Type Systems.

The basic idea motivating this use can be traced back to the earliest known translation from classical logic to intuitionistic logic due to Kolmogorov [7]. This translation was actually a translation of classical logic into minimal logic: the rule "ab falso quodlibet" is never used, and the absurd proposition $\perp$ in Kolmogorov's paper can thus be replaced formally by any proposition $A$. 
Kolmogorov saw the use of his translation as the development of "pseudomathematics," where, intuitively speaking, all notions and all lemmas occurring in a proof are defined and proved "relatively to a fixed proposition $A$ ".

This is this feature of $A$-translation that we use essentially here. In general, it is hard to see how to transform a paradox into a looping combinator. Howe's argument [6] is rather involved, done with computer assistance, and shows only how to extract a looping combinator out of one specific paradox. Our approach is more general. We show how to build a looping combinator from any given paradox. Indeed, when we apply $A$-translation to a paradox, we get a proof of $A$ where all notions and lemmas are defined and proved "relatively to $A$ ". This proof is then transformed without too much problems into a looping combinator.

The first section defines a class of "logical" Pure Type Systems in which we will define an $A$-translation. The second section describes the $A$-translation for logical Pure Type Systems. We state then a significant property of proofs obtained from the $A$-translation in the third section. This property is exploited to show the existence of a looping combinator in inconsistent Type Systems. The last section gives some examples of Type Systems containing looping combinators. We end by raising some questions suggested by our work.

\section{Logical Pure Type Systems}

We use here the standard definition of Pure Type Systems from Barendregt and Geuvers-Nederhof $[1,4]$. In particular, we make fairly heavy implicit use of the general properties of Pure Types Systems as presented in [4].

Definitions : A Pure Type System $L$ is logical iff it is functional (see [4]) and contains two distinguished sorts Prop and Type such that

- Prop : Type is an axiom of $L$

- (Prop, Prop, Prop) is a rule of $L$

- There are no sorts of type Prop

In a logical Pure Type System, the terms of type Prop are called propositions, and the terms of type a proposition are called proofs.

Definition : A logical Pure Type System is inconsistent iff there exists a proof of $A$ in the context $A:$ Prop.

Definition : A logical Pure Type System is said to be nondependent iff the only rules concerning Prop are of the form (S, Prop, Prop) where $S$ is some sort. 
Remark : simply-typed $\lambda$-calculus, system $F, F_{\omega}$ (see [4]) are nondependent logical Pure Type Systems. On the other hand, a type system with a type of all types, with Prop = Type is not logical because Prop is then a sort of type Prop. The calculus of constructions, see [4], is logical, but is not nondependent because it has the rule (Prop, Type, Type).

Lemma 1 In a nondependent logical Pure Type System, if $X=\left(X_{1} X_{2}\right)$ and $X_{1}$ or $X_{2}$ is a proof, then $X$ is a proof.

Proof There exist $Y_{1}, Y_{2}, S_{1}, S_{2}$ and $S$ such that $X_{1}:\left(x_{2}: Y_{2}\right) Y, X_{2}: Y_{2}$, $Y: S, Y_{2}: S_{2}$ and $\left(S_{2}, S, S_{1}\right)$ is a rule. If $X_{1}$ is a proof, then $S_{1}=$ Prop and so $S=$ Prop. If $X_{2}$ is a proof, then $S_{2}=$ Prop, and so $S_{1}=S=$ Prop.

Lemma 2 In a nondependent logical Pure Type System, if $Y$ is a subterm of $X$ and $Y$ is a proof, then $X$ is a proof.

Proof

By induction on the term $X$. We can as well assume that $Y$ is a subterm of $X$ distinct from $X$.

In such a case, the term $X$ cannot be a variable, a constant.

if $X$ is $\lambda x: X_{1} \cdot X_{2}$ then, by induction hypothesis, since $X_{1}$ is not a proof, $Y$ is a subterm of $X_{2}$, and hence by induction hypothesis, $X_{2}$ is a proof. Hence $X$ is a proof.

if $X$ is $\left(X_{1} X_{2}\right)$ then by induction hypothesis, $X_{1}$ or $X_{2}$ is a proof. By lemma 1 , this implies that $X$ is a proof.

The case where $X$ is a product is impossible by induction hypothesis.

Remark : This lemma implies that if $C:$ Prop in a context containing the declaration of a proof variable $h: B$, then $h$ is not a subterm of $C$. Thus, any product $\Pi h:$ B.C built from the rule (Prop, Prop, Prop) is nondependent and can be written $B \rightarrow C$.

Lemma 3 Let $L$ be nondependent logical Pure Type System and $p$ a proof in a context $\Gamma$.

Then $p$ is either a variable of the context, or a constant, or $\lambda x: Y . q$ where $q$ is a proof in $\Gamma, x: Y$, or $(q X)$ where $q$ is a proof in $\Gamma$.

Proof Direct by case analysis.

\section{A-translation in nondependent logical Pure Type Systems}

In all the section, we assume to be in a fixed nondependent logical Pure Type System, and inside the context $A$ : Prop. 
Notation: Let $B$ be a proposition. We will write $[B]$ for the proposition $(B \rightarrow A) \rightarrow A$.

We now define a translation ${ }^{+}$on terms which are not proofs. This translation depends on the type of the subterms, and it is defined relatively to a context in which the term is well-formed. Notice that it is not clear a priori that $M^{+}$is a well-formed term, so that a priori $M^{+}$is defined only as a pseudo-term (see [4].) Proposition 1 will later show that $M^{+}$is actually a well-formed term.

Definition : Let $X$ be a well-formed term in the context $\Gamma$, different from a proof.

- $X^{+}$is $X$ if $X$ is a variable, a constant or a sort

- $\left(X_{1} X_{2}\right)^{+}$is $\left(X_{1}^{+} X_{2}^{+}\right)$

- $\left(\lambda x: X_{1} \cdot X_{2}\right)^{+}$is $\lambda x: X_{1}^{+} \cdot X_{2}^{+}$

where $X_{2}^{+}$is defined in $\Gamma, x: X_{1}$

- the definition of $\left(\Pi x: X_{1} \cdot X_{2}\right)^{+}$depends on the type of $X_{2}$ and $X_{1}$ :

if $X_{2}$ is a proposition $B_{2}$ in $\Gamma$

then if $X_{1}$ is a proposition $B_{1}$ in $\Gamma$

then $\left(B_{1} \rightarrow B_{2}\right)^{+}$is $\left[B_{1}^{+}\right] \rightarrow\left[B_{2}^{+}\right]$

else $\left(\Pi x: X_{1} \cdot B_{2}\right)^{+}$is $\Pi x: X_{1}^{+} \cdot\left[B_{2}^{+}\right]$,

where $B_{2}^{+}$is defined in $\Gamma, x: X_{1}$

else $\left(\Pi x: X_{1}, X_{2}\right)^{+}$is $\Pi x: X_{1}^{+} \cdot X_{2}^{+}$

where $X_{2}^{+}$is defined in $\Gamma, x: X_{1}$

Remark : lemma 3 justifies the previous definition by cases.

Lemma 4 For any terms $X$ well-formed in $\Gamma, y: Y$ and $Z$ well-formed in $\Gamma$ different from proofs, then $(X[y:=Z])^{+}$is identical to $X^{+}\left[y:=Z^{+}\right]$.

Proof It is straightforward.

Lemma 5 For any terms $X$ and $Y$ well-formed in $\Gamma$ different from proofs, $X={ }_{\beta} Y$ implies $X^{+}={ }_{\beta} Y^{+}$.

Proof It suffices to prove that $\left(\lambda z: Z . Z^{\prime} Z^{\prime \prime}\right)^{+}$reduces to $\left(Z^{\prime}\left[z:=Z^{\prime \prime}\right]\right)^{+}$. This follows from lemma 4.

We now define a translation * on propositions and contexts

Definitions : Let $B$ be a proposition in a certain context, $B^{*}$ is defined as $\left[B^{+}\right]$. Let $\Gamma$ be a well-formed context, $\Gamma^{*}$ is defined inductively like this : 
- if $\Gamma$ is the empty context then $\Gamma^{*}$ is the empty context

- if $\Gamma$ is $\Gamma^{\prime}, x: X$, where $X$ is not a proposition then $\Gamma^{*}$ is $\Gamma^{\prime *}, x: X^{+}$

- if $\Gamma$ is $\Gamma^{\prime}, h: B$, where $B$ is a proposition then $\Gamma^{*}$ is $\Gamma^{\prime *}, h: B^{*}$

Lemma 6 For any propositions $B$ and $C$ in $\Gamma, B={ }_{\beta} C$ implies $B^{*}={ }_{\beta} C^{*}$.

Proof Straightforward by lemma 5 .

Proposition 1 If $\Gamma \vdash X: Y$ and $X$ is not a proof then $\Gamma^{*} \vdash X^{+}: Y^{+}$. If $\Gamma \vdash B:$ Prop then $\Gamma^{*} \vdash B^{*}:$ Prop.

Proof We prove this simultaneously by induction on the structure of the derivation of $\Gamma \vdash X: Y$ (resp. $\Gamma \vdash B:$ Prop.) The case of conversion is done by lemma 5 . Lemma 2 assures us that the derivation of $\Gamma \vdash X: Y$ (resp $\Gamma \vdash B:$ Prop) encounters no proofs.

Lemma 7 For any propositions $B$ and $C$ in $\Gamma$, if $\Gamma^{*} \vdash p: B^{*}$ and $B={ }_{\beta} C$ then $\Gamma^{*} \vdash p: C^{*}$.

Proof By lemma 6 and the conversion rule in Pure Type Systems. Proposition 1 assures that $\Gamma^{*} \vdash C^{*}:$ Prop.

We now define translation ${ }^{*}$ on proofs. As for the translation ${ }^{+}$, it is defined relatively to a context in which the term is a well-formed proof $p$, and it is not clear a priori that $p^{*}$ is a well-formed term, so that $p^{*}$ is defined only as a pseudo-term. Theorem 1 will actually show that $p^{*}$ is indeed a well-formed term which is a proof.

Definition : Let $p$ be a proof in the context $\Gamma$.

- $p^{*}$ is $p$ if $p$ is a variable or a constant

- if $p$ is $\lambda h: B . q$, with $B$ a proposition, and $q: C$, then $p^{*}$ is $\lambda k:\left(\left(B^{*} \rightarrow C^{*}\right) \rightarrow A\right) .\left(k \quad \lambda h: B^{*} \cdot \lambda k^{\prime}:\left(C^{+} \rightarrow A\right) \cdot\left(q^{*} k^{\prime}\right)\right)$

where $q^{*}$ is defined in $\Gamma, h: B$

- if $p$ is $\lambda x: Y . q$, with $Y$ not a proposition, and $q: C$, then $p^{*}$ is $\lambda k:\left(\left(\Pi x: Y . C^{*}\right) \rightarrow A\right) .\left(k \lambda x: Y . \lambda k^{\prime}:\left(C^{+} \rightarrow A\right) \cdot\left(q^{*} k^{\prime}\right)\right)$

where $q^{*}$ is defined in $\Gamma, x: Y$

- if $p$ is $\left(p_{1} p_{2}\right)$ and $p_{1}: B \rightarrow C$ then $p^{*}$ is $\lambda k:\left(C^{+} \rightarrow A\right) \cdot\left(p_{1}^{*} \quad \lambda h_{1}:\left(B^{*} \rightarrow C^{*}\right) \cdot\left(h_{1} \quad p_{2}^{*} \quad k\right)\right)$ 
- if $p$ is $\left(p_{1} X\right)$, when $X$ is not a proof, and $p_{1}: \Pi x: Y . C$, then $p^{*}$ is $\lambda k:\left(C[x:=X]^{+} \rightarrow A\right) \cdot\left(p_{1}^{*} \quad \lambda h_{1}:\left(\Pi x: Y^{+} . C^{*}\right) \cdot\left(h_{1} X^{+} k\right)\right)$

Remark : lemma 3 justifies the previous definition by cases.

Theorem 1 Let $B$ be a proposition in $\Gamma$. If $\Gamma \vdash p: B$ then $\Gamma^{*} \vdash p^{*}: B^{*}$

Proof By induction on the structure of the derivation of $\Gamma \vdash p: B$. The case of proposition conversion is done by lemma 7. Proposition 1 treats the case of judgements $\Gamma \vdash X: Y$ with $X$ not a proof.

Remark 1: ${ }^{*}$ is a Kolmogorov-like $A$-translation. It generalizes an $A$-translation of Paulin-Mohring [11] for the Calculus of Constructions with data types distinguished from propositions, and is inspired by a classical/intuitionistic translation of Girard [5] for higher order $\lambda$-calculi.

Remark 2: if we assume Church-Rosser property for the Pure Type System we are considering, lemma 5 holds also for $\beta \eta$-conversion and therefore proposition 1 and theorem 1 still hold in presence of $\beta \eta$-conversion. However, ChurchRosser property for general Pure Type Systems (not necessarilly normalisable) with $\beta \eta$-conversion seems still to be an open problem.

\section{Long $A$-applicativity}

As we said in the introduction, the original motivation in using $A$-translation was the fact that, intuitively, proofs that we get by $A$-translation "proves only A." Trying to make precise this remark leads to the following notion.

Definition : The notion of long $A$-applicative proof in a context $\Gamma$ is defined by the following cases:

- the variable $h$ of type $B$ with $B:$ Prop is a long $A$-applicative proof if $h: B$ is in $\Gamma$

- $\lambda x_{1}: Y_{1} \ldots \lambda x_{n}: Y_{n} . p$ is a long $A$-applicative proof in $\Gamma$ if $p$ is a long $A$-applicative proof in $\Gamma, x_{1}: Y_{1}, \ldots, x_{n}: Y_{n}$ and if $p$ is of type $A$

- $(p q)$ is a long $A$-applicative proof in $\Gamma$ if $p$ and $q$ are long $A$-applicative proofs in $\Gamma$.

- $(p X)$ where $X$ is not a proof is a long $A$-applicative proof in $\Gamma$ if $p$ is a long $A$-applicative proof in $\Gamma$.

Proposition 2 If $p$ is a proof in $\Gamma$ then $p^{*}$ is long A-applicative in $\Gamma^{*}$.

Proof Direct from the definition of $p^{*}$. 
Lemma 8 If $p$ is a long A-applicative proof in a context $\Gamma, h: B$ and $q$ is long $A$-applicative in $\Gamma$ then $p[h:=q]$ is long A-applicative in $\Gamma$.

If $p$ is a long $A$-applicative proof in a context $\Gamma, x: Y$ and $X$ is not a proof in $\Gamma$ then $p[x:=X]$ is long A-applicative in $\Gamma$.

Proof By induction on the structure of $p$.

\section{Looping combinators}

The idea of Meyer and Reinhold [9] to obtain a recursion combinator in the inconsistent system Type : Type was to exploit the non normalisability of the proof of the inconsistency by inserting some " $f$ " in it in order to obtain a term $p_{0}$ such that $p_{0}$ reduces to $\left(f p_{1}\right)$ and then $p_{1}$ to $\left(f p_{2}\right)$, and so on... From such a sequence, it is direct to build a family of terms $Y_{n}: \Pi A:$ Type. $(A \rightarrow A) \rightarrow A$ such that $\left(Y_{n} A f\right)=f\left(Y_{n+1} A f\right)$.

Definition : Let $T$ be a Pure Type System and $S$ a sort of $T$. A looping combinator of sort $S$ in $T$ is a term $Y: \Pi A: S .(A \rightarrow A) \rightarrow A$ such that there exists a sequence of terms $Y_{0} \equiv Y, Y_{1}, \ldots Y_{n} \ldots$, of type $\Pi A: S .(A \rightarrow A) \rightarrow A$ such that for any $A: S, f: A \rightarrow A$

$\left(Y_{n} A f\right)=\beta f\left(Y_{n+1} A f\right)$

Howe [6] applied the same idea to transform the paradox of Girard [5] into a looping combinator by a direct mechanichal analysis of the term corresponding to this paradox.

We are now going to show how to build a looping combinator in any inconsistent nondependent logical Pure Type System. The last section will show that this implies in particular the existence of a looping combinator also for Type : Type.

From now on, we assume to be in a fixed inconsistent nondependent logical Pure Type System, and inside the context A : Prop.

Proposition 3 There exists a long A-applicative proof of $A$.

Proof Since the type system is inconsistent, there exists a proof $q_{A}$ of $A$ in the context $A$ : Prop. By theorem $1,\left(q_{A}\right)^{*}$ is a proof of $A^{*}$ in the context $A:$ Prop and by proposition 2 , this proof is long $A$-applicative. But $A^{*}$ is $(A \rightarrow A) \rightarrow A$, and $p_{A}=\left(\left(q_{A}\right)^{*} \lambda x: A . x\right)$ is a long $A$-applicative proof of $A$.

We now precise what kind of term is a long $A$-applicative proof of $A$ :

Lemma 9 A long A-applicative proof of $A$ is of the following form:

$$
\left(\left(\lambda x^{1}: Y^{1} \ldots \lambda x^{m}: Y^{m} \cdot q\right) X^{1} \ldots X^{m}\right)
$$


with $m \geq 1, q: A$ and each $X^{i}$ is either long A-applicative or not a proof.

Proof Let $p$ be a long $A$-applicative proof of $A$ in the context $A$ : Prop. Since $A$ is atomic, $A$ cannot be convertible to a product by Church-Rosser. Hence, by uniqueness of type, $p$ does not begin with an abstraction.

Therefore it is of the following form:

$$
\left(p^{\prime} X^{1} \ldots X^{m}\right) \text { with } m \geq 0 \text { and } p^{\prime} \text { either a variable or an abstraction }
$$

Since we are in the context $A:$ Prop $p^{\prime}$ cannot be a variable, $m \geq 1$ and $p^{\prime}$ begins with an abstraction. And since $p$ is long $A$-applicative, $p^{\prime}$ is of the following form:

$$
\lambda x^{1}: Y^{1} \ldots \lambda x^{m^{\prime}}: Y^{m^{\prime}} \cdot q \text { with } m^{\prime} \geq 1 \text { and } q: A .
$$

The type of $q$ remains $A$ by instantiation, hence $m$ cannot be greater than $m^{\prime}$. And since $p$ proves $A, m^{\prime}$ cannot be greater than $m$. Hence we have $m=m^{\prime}$, i.e. $p$ has the desired form.

We now define a strategy of reduction applicable to long $A$-applicative proofs of type $A$.

Definition : Let $p$ be a long $A$-applicative proofs of type $A$. By lemma $9, p$ is

$$
\left(\left(\lambda x_{1}: Y_{1} \ldots \lambda x_{n}: Y_{n} . q\right) X_{1} \ldots X_{n}\right) \text {, with } n \geq 1 \text { and } q: A \text {, }
$$

$\operatorname{red}(p)$ is then the following term of type $A$

$$
q\left[x_{1}:=X_{1}\right] \ldots\left[x_{n}:=X_{n}\right] .
$$

Lemma 10 For any long $A$-applicative proof $p$ of $A$ in $A: \operatorname{Prop}$, red $(p)$ is a long A-applicative proof of $A$ in $A$ : Prop.

Proof By lemma 8.

We now define the transformation $p^{f}$ which inserts "marks" inside long $A$ applicative proofs $p$ in such a way that for any long $A$-applicative proofs $p$ of $A$ $\operatorname{red}\left(p^{f}\right)$ is $\left(f(\operatorname{red}(p))^{f}\right)$.

Definition : Let $p$ be a long $A$-applicative proof in a context $\Gamma$

$p^{f}$ is defined inductively in the context $\Gamma, f: A \rightarrow A$ as follows:

- if $p$ is a variable $h$ in $\Gamma$ then $p^{f}$ is $h$ in $\Gamma$,

- if $p$ is $\lambda x_{1}: Y_{1} \ldots \lambda x_{n}: Y_{n} . q$ in $\Gamma$ then $p^{f}$ is $\lambda x_{1}: Y_{1} \ldots \lambda x_{n}: Y_{n} \cdot\left(f q^{f}\right)$ in $\Gamma$ where $q^{f}$ is defined in $\Gamma, f: A \rightarrow A, x_{1}: Y_{1}, \ldots, x_{n}: Y_{n}$,

- if $p$ is $\left(p_{1} p_{2}\right)$ then $p^{f}$ is $\left(p_{1}^{f} p_{2}^{f}\right)$, 
- if $p$ is $\left(p_{1} M\right)$ with $M$ not a proof, then $p^{f}$ is $\left(p_{1}^{f} M\right)$.

Remark : $p^{f}$ is of same type as $p$ and is long $A$-applicative also.

Lemma 11 If $p$ is an A-applicative proof in the context $\Gamma, h: B$ and $q$ an A-applicative proof of $B$ in $\Gamma$ then $p^{f}\left[h^{\prime}:=q^{f}\right]$ is $(p[h:=q])^{f}$.

If $p$ is an A-applicative proof in the context $\Gamma, R: T$ and $M: T$ not a proof then $p^{f}[R:=M]$ is $(p[R:=M])^{f}$.

Proof By structural induction on $p$ and by lemma 8

Lemma 12 For any long A-applicative proof $p$ of $A$, red $\left(p^{f}\right)$ is $\left(f(\operatorname{red}(p))^{f}\right)$.

Proof $p$ is of the form

$$
\left(\left(\lambda x^{1}: Y^{1} \ldots \lambda x^{m}: Y^{m} \cdot q\right) X^{1} \ldots X^{m}\right)
$$

and then $p^{f}$ is

$$
\left(\left(\lambda x^{1}: Y^{1} \ldots \lambda x^{m}: Y^{m} \cdot\left(f q^{f}\right)\right)\left(X^{1}\right)^{f} \ldots\left(X^{m}\right)^{f}\right),
$$

which reduces by lemma 11 to $\left(f(\operatorname{red}(p))^{f}\right)$.

Lemma 13 There exists a sequence of terms $M_{0}, M_{1}, \ldots, M_{n}, \ldots$ defined in the context $A:$ Prop $f: A \rightarrow A$ such that $M_{n}={ }_{\beta}\left(f M_{n+1}\right)$.

Proof We define a sequence of terms $p_{n}$ as follows. First we define $p_{0}$ to be any long $A$-applicative proof of $A$ in the context $A:$ Prop, using proposition 3 . We then define $p_{n+1}$ to be $\operatorname{red}\left(p_{n}\right)$. Each proof term $p_{n}$ is long $A$-applicative proof of $A$ in $A$ : Prop by lemma 10 .

Let $M_{n}$ be $p_{n}^{f}$. The sequence $M_{0}, \ldots, M_{n}, \ldots$ satisfies lemma 13 by lemma 12 .

Theorem 2 In any inconsistent nondependent logical Pure Type System, there exists a looping combinator of type Prop.

Proof Direct from lemma 13

Remark : The proof given here is constructive. We can effectively transform any proof of $A$ in the context $A$ : Prop into a looping combinator. 


\section{Applications}

We describe here the systems $U^{-}, U$ and Type : Type as Pure Type Systems.

The system $U^{-}$is the Pure Type System defined by the following sorts:

Prop, Type and Class,

the axioms:

Prop : Type and Type: Class

and the rules:

$$
\begin{aligned}
& \text { (Prop, Prop, Prop) } \\
& \text { (Type, Prop, Prop) } \\
& \text { (Type, Type, Type) } \\
& \text { (Class, Type, Type). }
\end{aligned}
$$

System $U$ is the same as system $U^{-}$plus the following rule:

$$
\text { (Class, Prop, Prop) }
$$

The system Type: Type is the Pure Type System with the only sort:

$$
\text { Type }
$$

the only axiom:

$$
\text { Type : Type }
$$

and the only rule:

$$
\text { (Type,Type, Type) }
$$

Both systems $U$ and $U^{-}$are nondependent logical Pure Type System. They are both inconsistent, as shown in $[2,5]$. Hence, by theorem 2, they contain a looping combinator of sort Prop. It is clear that a looping combinator for one of this system translates directly in a looping combinator of sort Type for Type : Type.

Here is a direct application. Call a nondependent logical type system impredicative iff it contains the rule (Type, Prop, Prop). 
Theorem 3 Convertibility is undecidable for inconsistent impredicative logical Pure Type System. Furthermore, convertibility and type-checking is undecidable for Type : Type.

Proof The arguments of [9], which assumed the existence of a fixed-point combinator, apply directly using a looping combinator instead.

For sake of completeness, we include a sketch of these arguments. First, it is standard [5] how to represent primitive recursive functions as terms of type $N \rightarrow N$, where $N$ is the proposition $\Pi X . X \rightarrow(X \rightarrow X) \rightarrow X$, and the number $n$ is represented by the term $\lambda X \cdot \lambda x \cdot \lambda f .\left(f^{n} x\right)$. A looping combinator family allows the numeralwise representation of any partial recursive function $\phi$ by a term $\Phi$ : namely $\Phi t_{n}={ }_{\beta} t_{k}$ iff $\phi(n)=k$. This entails the undecidablity of convertibility in any inconsistent impredicative logical Pure Types System.

The same reasoning will apply to Type : Type by taking $N$ to be the type $\Pi X . X \rightarrow(X \rightarrow X) \rightarrow X$. Furthermore, in this case the problem whether $\phi(n)=0$ reduces to the question whether $(f x)$ is typable in the context $P$ : $N \rightarrow$ Type, $f: P\left(t_{0}\right) \rightarrow N, x: P\left(\Phi\left(t_{n}\right)\right)$. Likewise, checking specific type judgements is undecidable, since $\phi(n)=0$ reduces to the question whether $x$ has type $P\left(\Phi\left(t_{n}\right)\right)$ in the context $P: N \rightarrow$ Type, $x: P\left(t_{0}\right)$.

Notice however that the normalisation theorem for system $F$ [5] implies directly the decidability of type-checking for the system $U^{-}$and the system $U$.

\section{Conclusion}

We would like to raise some problems:

- The problem of the existence of a fixed-point combinator for the system Type : Type is still open.

- Is it possible to derive the existence of a looping combinator from the existence of a paradox in a more direct way than by using $A$-translation?

- For the system $U^{-}$it is possible to define a "stripping" operation that associates to any proof term the untyped $\lambda$-term we get by forgetting the type information. We conjecture that the usual direct proof of non typability of the term $\left(\lambda x\left(\begin{array}{lll}x & x\end{array}\right) \lambda x(x x)\right)$ in system $F$ extends to show that this term is not typable in system $U^{-}$.

\section{Acknowledgements}

The authors want to thank Herman Geuvers, Chet Murthy, Erik Palmgren and Benjamin Werner both for their remarks about the paper and for enjoyable discussions about $A$-translation and Pure Type Systems. Thanks also to Henk Barendregt for his definition of looping combinators. 


\section{References}

[1] H. Barendregt. Introduction to Generalized Type System. In the Journal of Functional Programming, Volume 1, Part 2, April 1991, pages 125 - 154.

[2] T. Coquand. A New Paradox in Type Theory. To appear in the Proceedings of the 9th International Congress of Logic, Methodology and Philosophy of Science, Uppsala, August 1991.

[3] H. Friedman. Classically and Intuitionistically Provably Recursive Functions. In Higher Set Theory, ed. G.H. Müller and D.S. Scott. Springer Verlag Lecture Notes 669, 1978, pages 21 - 27.

[4] H. Geuvers and M.-J. Nederhof. Modular proof of strong normalisation for the calculus of constructions. In the Journal of Functional Programming, Volume 1, Part 2, April 1991, pages 155 - 189.

[5] J.Y. Girard. Interprétation fonctionnelle et élimination des coupures dans l'arithmétique d'ordre supérieur. Thèse de doctorat d'état de l'université Paris 7, 1972 .

[6] D. J. Howe. The computational behaviour of Girard's paradox. In Proceedings of the Second Symposium of Logic in Computer Science (Ithaca, N.y.), IEEE, 1987, pages 205 - 214.

[7] A.N. Kolmogorov. On the principle of excluded middle. 1925. in From Frege to Gödel : a source book in mathematical logic, 1879-1931. J. Van Heijenoort. Harvard University Press, 1967.

[8] D. Leivant. Syntactic translations and provable recursive functions. Journal of Symbolic Logic 50, 1985, pages $682-688$.

[9] A. R. Meyer, M. B. Reinhold. "type" is not a type. In Conference record of the thirteenth annual ACM symposium on principles of programming languages, Association for Computing Machinery, SIGACT, SIGPLAN, 1986, pages $287-295$.

[10] C. Murthy Extracting Constructive Content From Classical Proofs. Ph.D. Thesis, Cornell University, 1990.

[11] C. Paulin Extraction de programmes dans le Calcul des Constructions. Thèse de doctorat de l'université Paris 7, 1989. 